\title{
Mechanisms of cancer metastasis to the bone
}

\author{
Juan Juan YIN, Claire B. POLLOCK, Kathleen KELLY* \\ Cell and Cancer Biology Branch, NCI, NIH, Bethesda, MD 20892, USA
}

\begin{abstract}
Some of the most common human cancers, including breast cancer, prostate cancer, and lung cancer, metastasize with avidity to bone. What is the basis for their preferential growth within the bone microenvironment? Bidirectional interactions between tumor cells and cells that make up bone result in a selective advantage for tumor growth and can lead to bone destruction or new bone matrix deposition. This review discusses our current understanding of the molecular components and mechanisms that are responsible for those interactions.
\end{abstract}

Keywords: cancer, bone metastases, osteolytic metastasis, osteoblastic metastasis.

\section{INTRODUCTION}

Bone metastases are a frequent complication of certain cancers including breast and prostate cancers with more frequency, and lung and kidney with less frequency[1]. Greater than $50 \%$ of patients with advanced breast or prostate cancer show bone metastases. Importantly, once tumors metastasize to bone, they are virtually incurable and result in significant morbidity prior to a patient's death [2, 3]. Bone metastases can lead to pain, pathological fractures, nerve compression syndromes, and hypercalcemia. Current treatments are mainly palliative. New therapeutics are needed, and a greater understanding of bone tropic tumor cells, especially those properties that lead to their successful growth within the bone microenvironment is one path to devise innovative approaches.

Why do certain cancers metastasize so readily to bone? Osteotropic cancers are a specific example of the more general observation that primary tumors show organspecific patterns of metastasis. In 1889, Stephen Paget set forth the "seed and soil" hypothesis, which suggests that the interplay between the properties of cancer cells and the particular organ microenvironment determines the selective advantage of cells to grow [4]. More specifically, the ability of cells to survive, clonally expand, and recruit a blood supply is expected to determine successful metastasis. Other factors thought to play a role in sitespecific metastasis are anatomic considerations such as

*Correspondence: Kathleen KELLY

E-mail: kkelly@helix.nih.gov blood flow patterns from the primary tumor and the homing ability of cancer cells for certain tissues. Homing to specific organs is likely regulated by chemoattractant factors and adhesion molecules that are produced by the target organ in combination with the proper counter-receptors expressed in the tumor cells $[5,6]$

An important consideration in understanding bone metastases is the unique composition of the "soil" or the bone microenvironment. Bone is a dynamic organ composed of cells of various embryonic origins including hematopoietic, stromal, endothelial, and other cell types [7]. Two cell types, osteoclasts and osteoblasts regulate bone modeling that occurs during development and bone remodeling that occurs in the adult [5]. Osteoclasts are derived from precursors in the mononuclear-phagocyte lineage and are responsible for bone resorption. Osteoblasts are derived from the stromal cell lineage and are responsible for laying down new bone matrix. A significant factor regulating bone remodeling is the direct interaction between osteoblasts and osteoclasts. The expression of RANK ligand (RANKL) on the surface of osteoblasts engages the receptor, RANK, on osteoclast precursors, leading to their maturation. In addition, a plethora of systemic and locally acting factors derived from endocrine, immune, and other systems can impact osteoclast and osteoblast function [1] (see Tab. 1 and Fig. 1). Such factors can enhance the activity of osteoclasts, either indirectly through stimulating the expression of RANKL on osteoblasts, or through direct effects on osteoclast and osteoblast function. Importantly, one predominant source of locally-acting factors is the one released from degraded bone matrix. 
Bone metastases are generally characterized as osteolytic, leading to bone destruction, or osteosclerotic (osteoblastic), leading to new bone formation [3]. Breast cancer metastases are usually osteolytic, and prostate cancer metastases are usually osteoblastic. The type of metastasis is a reflection of the local interaction between tumor cells and the bone remodeling system. Thus, the development of osteolytic and osteoblastic lesions results from a functional interaction between tumor cells and osteoclasts or osteoblasts, respectively. As a result of the dynamic nature of bone

Tab. 1 Soluble factors influencing osteoblast and osteoclast function and metastatic cancer growth in the bone

\begin{tabular}{cl}
\hline Osteolytic factors & Osteoblastic factors \\
\hline IL-6 & Endothelin 1 \\
PTHrP & BMPs \\
Prostaglandins & Prostaglandins \\
TNF $\alpha$ & TNF $\alpha$ \\
EGF, TGF $\alpha$ & IGFs \\
TGF $\beta$ & TGF $\beta$ \\
IL-1 & IL-1 \\
Colony Stimulating Factors & BDGF \\
PDGF & PDGF \\
\hline
\end{tabular}

remodeling and the potential heterogeneity of metastatic lesions, patients can have both osteolytic and osteosclerotic lesions or mixed lesions containing both elements. In addition, due to the regulatory interactions between osteoclasts and osteoblasts, secondary bone formation occurs in response to bone destruction.

\section{OSTEOLYTIC METASTASIS}

Osteolytic metastasis is the most common form of bone metastasis in all cancer patients. The dominant lesion is lytic and destructive, although local bone formation response can also be observed. Osteolytic metastasis occurs in solid tumors including breast cancer, prostate cancer, thyroid cancer, lung cancer, and renal cancer. In addition, multiple myeloma typically causes extensive bone destruction [3]. Most in vivo studies indicate that osteolysis is caused by osteoclast stimulation, not by the direct effects of cancer cells on bone. Osteolytic metastases are associated with increased osteoclast activity and reduced osteoblast activity that is uncoupled from bone resorption.

It is becoming evident that osteolytic bone metastases are derived from a complex cycle of progressive interactions between tumor cells and the bone microenvironment, which has been called a "viscous cycle" [8]. Characteristics that allow tumor cells to localize to bone, tumor derived-

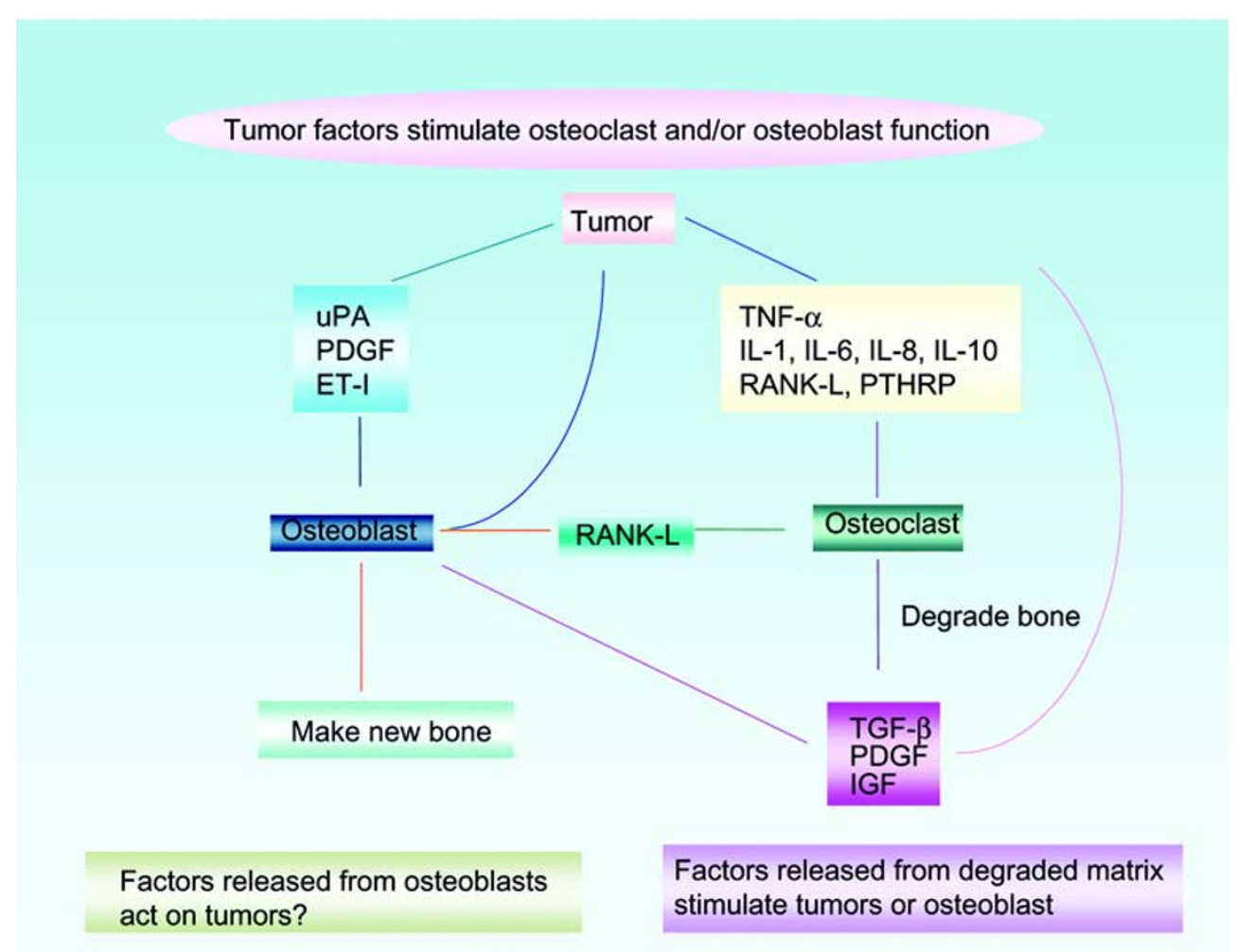

Fig. 1 Components that contribute to bone metastasis growth via a vicious cycle. 
factors that stimulate responses from osteoclasts and osteoblasts, and stored factors within the bone matrix contribute to this cycle (See Fig. 1).

Tumor cells produce chemokine receptors, cell adhesion molecules, and cell surface receptors that enable them to attach to the bone matrix and establish growth in bone. CXCR4 is highly expressed in human breast cancer cells, malignant breast tumors and metastases. CXCR4 ligand CXCL12/SDF-1 $\alpha$ is abundant in bone marrow stromal cells [9]. A recent study showed that CXCR4 together with other factors including CTGF, IL-11 and OPN promote osteolytic bone metastasis in MDA-MB-231 breast cancer cells [10]. Bone stromal cells express VCAM- 1 and type I collagen. Tumor cells that express $\alpha_{4} \beta_{1}$ or $\alpha_{2} \beta_{1}$ integrins may preferentially adhere to bone.

A specific type of osteolytic metastasis is cortical metastasis, which is most commonly seen in lung and renal cancer patients and may be particularly relevant for the later stage of disseminated disease. This type of lesion may occur more frequently than previously expected. A cortical metastasis can develop and destroy the cortex without any involvement of the medullary cavity. The mechanism of cortical metastasis is not well studied, but an important consideration is the capillary system of the cortex, which likely plays a significant role in the development of cortical metastasis. The cortex receives its vascular supply from anastomatic branches including medullary, periosteal and nutrient artery vessels. Arterial disseminated metastases can be transported to an intracortical location from any one of the three vessel sources noted above [11, $12,13]$.

Tumor cells produce factors that stimulate osteoclastic bone resorption directly or indirectly. These factors include PTHrP, IL-1, IL-6, prostaglandin E2, TNF, and CSF-1. PTHrP is one of the major mediators of breast cancer related osteolytic bone metastasis. Patients with PTHrP positive breast cancer are more likely to develop bone metastases. PTHrP expression by breast cancer bone metastases $(92 \%)$ is greater than that at the primary sites $(50 \%)$ or nonbone metastases (17\%) [14]. These clinical observations have been confirmed by using a mouse model in which neutralizing antibody of PTHrP inhibited the development and progression of breast cancer related bone metastasis in vivo. IL-6 is constitutively expressed by renal, bladder, prostate, cervical, glioblastoma and breast carcinoma cells and immunohistochemical studies have demonstrated IL-6 expression in the cytoplasm of colon, prostate and breast carcinoma cells $[15,16,17,18]$. IL-6 is a potent stimulator of osteoclast formation and can enhance the effects of PTHrP on osteoclasts [19, 20]. Breast cancer cells also produce IL-1, TNF and prostaglandins which increase RANKL expression and stimulate osteoclasts [21,
22]. A recent study showed that breast cancer cells could stimulate survival of osteoclastogenesis and prolong osteoclast survival by expressing and secreting CSF-1[23, 24].

The bone microenvironment is rich in growth factors. During bone formation, osteoblasts lay down a variety of growth factors, which are incorporated into the bone matrix along with type I collagen. The process of bone resorption releases and/or activates these factors into the bone microenvironment. Many of these growth factors, including TGF $\beta$, FGFs, IGFs and BMP-2 not only are able to stimulate the growth of metastatic cancer cells in bone, but also stimulate the production and release of bone resorbing factors from tumor cells. Although TGF $\beta$ is a key negative growth regulator of normal breast and prostate cells, it acts as a tumor promoter in advanced cancers. TGF $\beta$ stimulates PTHrP production in a renal cell carcinoma, a squamous cell carcinoma, and a human breast adenocarcinoma, MDA-MB-231. BMP2 has been shown to stimulate cell growth, cell migration, and cell invasion of osteolytic prostate cancer PC-3 cells and osteoblastic prostate cancer LAPC-4 and LAPC-9.

Recently, there has been considerable improvement in our understanding of the successive steps by which tumor cells affect the bone. Among the tumor-produced factors, PTHrP is one of the major mediators of breast cancer related osteolytic bone metastasis. PTHrP binds to PTHR1 and induces the expression of RANKL on bone marrow stromal cells. RANKL binds the RANK receptor in osteoclast precursors and induces the formation of mature osteoclasts, which cause osteolytic bone resorption. TGF $\beta$ released from the bone microenvironment activates and stimulates cancer cells to produce more PTHrP, which in turn further activates osteoclastic bone resorption. Thus, tumor cells, tumor-produced PTHrP, osteoclasts and bone derived TGF $\beta$ form a vicious cycle to promote the development and progression of bone metastasis. Disruption of any step in the cycle may significantly decrease bone metastasis $[7,25]$.

\section{OSTEOBLASTIC BONE METASTASIS}

Some neoplasia produce bone metastases which are predominantly osteoblastic, most notably prostate cancer and approximately $15-20 \%$ breast cancers. Osteoblastic bone lesions are rare in other cancer types; however, they have been reported in, for example, colon, cervical and cervical cancer $[26,27,28]$. During osteoblastic bone metastases, the balance between bone resorption and bone formation is tipped in favor of the latter. Patients suffer severe bone pain and the poor quality of bone produced in osteoblastic bone metastases means that bone fractures are a common event. Models to investigate osteoblatic metastases are more 
rare than osteolytic metastases and the various mechanisms that play a role in osteoblastic metastases are still under definition. However, there are a number of protein candidates produced by cancer cells and which have been shown to enhance bone synthesis and increase bone density to result in osteoblastic lesions.

There is an accumulating body of evidence implicating Endothelin-1 (ET-1) as a central mediator of osteoblastic metastasis [29]. ET-1 was first identified as a potent vasoconstrictor and belongs to a family, which includes ET-2 and ET-3. Knockout studies indicated a role for ET-1 in bone formation since ET-1 null mice exhibit hypoplasia of the facial bones. ET-1 null mice die shortly after birth from respiratory failure and cardiac abnormalities [30]. Also serum ET-1 levels are increased in patients with bone osteoblastic lesions [31]. ET-1 mediates its effects on bone formation through the Endothelin $\mathrm{A}\left(\mathrm{ET}_{\mathrm{A}}\right)$ receptor. In a xenograft breast cancer model of osteoblastic bone metastasis, treatment with endothelin-1A-receptor antagonist reduced both osteoblastic bone metastases and tumor burden [32]. Osteoblastic prostate and breast cancers express ET1 and $\mathrm{ET}_{\mathrm{A}}$ receptor, suggesting that tumor produced ET-1 may have paracrine and autocrine effects. Indeed exogenous ET-1 increases prostate cancer cell proliferation and enhances the mitogenic effect of other growth factors including, IGF-1, PDGF and EGF [33]. The bone microenvironment may enhance ET-1 expression by cancer cells [34]. One mechanistic definition of ET-1 action includes the finding that ET-1 drives net bone formation by inhibiting osteoclast bone resorption and osteoclast motility [35]. Endothelin-1 receptor agonists are currently being tested in clinical trials. Statistical improvements have been reported with regard to bone pain levels, serum PSA levels, biologic bone markers of bone changes and bone metastases [36].

BMPs were originally characterized as inducers of bone formation at extraskeletal sites in vivo [37]. BMPs belong to the TGF $\beta$ superfamily and stimulate osteoblast differentiation through the activation of transcription factors, in particular Runx-2 [38]. In patients with prostate cancer BMP expression has been shown to correlate with increased recurrence rates and decreased survival [39]. BMP-4, -6 and 7 have been shown to be expressed by prostate cancer cells and in addition to their paracrine effects on osteoblasts, also play an important role in the survival and growth of the cancer cells themselves [40, 41]. Like TGF $\beta$, BMP appears to differentially affect early and advanced stage cancers [40]. Changes in the responsiveness of prostate cancer cells to BMPs may enable these cells to escape the growth inhibitory autocrine effects of BMPs and at the same time drive bone formation via BMPs paracrine effects on osteoblasts.

Proteases such as urokinase-type plasminogen receptor
(uPA) and Prostate Specific Antigen (PSA) are also implicated in osteoblastic bone metastasis. Overproduction of uPA by prostate cancer cells has been shown to increase osteoblastic bone metastasis in mice and antisense DNA to uPA was shown to reduce bone metastases by one third [ 42 , 43]. uPA can cleave and activate TGF $\beta$ which is produced in a latent form by osteoblasts. TGF $\beta$ regulates osteoblast and osteoclast differentiation but also regulates the growth of tumor cells themselves. PSA is a kallikrein serine protease, which is secreted by prostate cancer cells and used routinely as a marker of prostate cancer progression. PSA can cleave parathyroid hormone-related peptide (PTHrP). PTHrP is an important activator of osteoclast activation and therefore cleavage of PTHrP could block bone resorption. PSA may also activate osteoblast growth factors such as TGF $\beta$ [44].

Platelet-derived growth factor (PDGF) is also implicated in osteoblastic bone metastasis. PDGF is a dimeric polypeptide growth factor, forming $\mathrm{AA}, \mathrm{BB}$ and $\mathrm{AB}$ isoforms. The $\mathrm{BB}$ isoform is a potent osteotropic factor, stimulating the function of both osteoclasts and osteoblasts. In a mouse model of breast cancer bone metastasis, MCF7-Neu cells form osteoblastic bone metastases more aggressively than MCF-7 parentals and MCF-7/Neu mice were shown to have higher levels of serum PDGF-BB compared to $\mathrm{MCF}-7$ injected mice [45]. Conditioned media from $\mathrm{MCF}-7 / \mathrm{Neu}$ cells enhanced osteoblastic bone formation in organ cultures of neonatal mouse calvariae and neutralizing antibodies to PDGF-BB blocked this effect. Finally, introduction of human PDGF-B cDNA in MCF-7 cells, which produce osteolytic lesions, resulted in osteoblastic regions within osteolytic lesions.

There is evidence that osteoblastic metastasis also involves considerable osteolysis $[46,47]$. In prostate cancer patients with clinical osteoblastic lesions, blood and urinary levels of bone resorption markers are often elevated [48]. Clinical trials have suggested that blocking osteoclastic bone resorption reduces related skeletal events in prostate cancer patients [49]. In a mouse model of bone metastasis, osteoclasts numbers were shown to markedly increase at sites of early tumor invasion [47]. One theory as to why osteoclastogenessis is important for osteoblastic bone metastasis is that bone resorption by osteoclasts releases a variety of growth factors, which are stored as inactive forms in the bone matrix. The activation of growth factors may be required by cancer cells to maintain viability and proliferate and therefore in establishment of bone metastases.

\section{CONCLUSION}

Bone metastases are common in patients with advanced cancers, and their presence usually signifies serious mor- 
bidity and a grave prognosis. The unique environment in the bone and the variety of interactions that mediate cancer metastatic growth are just now being identified. Therapies exemplified by bisphosphonates that target general properties of bone metastases such as bone resorption are being evaluated [50]. In addition, more tailored therapies that are directed at specific factors such as PTHrP, endothelin, TGF $\beta$, or PDGF are being developed. A more detailed understanding of potential therapeutic targets are expected to be derived from the continued improvement of animal models and in vitro organ cultures, as well as the application of genomic technologies such as microarrays and proteomics to clinical bone metastastic samples.

\section{REFERENCES}

1 Mundy GR. Metastasis to bone: causes, consequences and therapeutic opportunities. Nat Rev Cancer 2002; 2:584-93.

2 Clark PE, Torti FM. Prostate cancer and bone metastases: medical treatment. Clin Orthop 2003, 415:S148-57.

3 Roodman GD. Mechanisms of bone metastasis. N Engl J Med 2004; 350: 1655-64.

4 Paget S. The distribution of secondary growths in cancer of the breast. Cancer Metastasis Rev 1989; 8:98-101.

5 Boyce BF, Yoneda T, Guise TA. Factors regulating the growth of metastatic cancer in bone. Endocr Relat Cancer 1999; 6:33347.

6 Vander Griend DJ, Rinker-Schaeffer CW. A new look at an old problem: the survival and organ-specific growth of metastases. Sci STKE 2004; 2004:pe3.

7 Guise TA, Mundy GR. Cancer and bone. Endocr Rev 1998; 19: 18-54.

8 Chirgwin JM, Guise TA. Molecular mechanisms of tumor-bone interactions in osteolytic metastases. Crit Rev Eukaryot Gene Expr 2000; 10:159-78.

9 Muller A, Homey B, Soto H, et al. Involvement of chemokine receptors in breast cancer metastasis. Nature 2001; 410:50-6.

10 Kang Y, Siegel PM, Shu W, et al. A multigenic program mediating breast cancer metastasis to bone. Cancer Cell 2003; 3:537-49.

11 Deutsch A, Resnick D. Eccentric cortical metastases to the skeleton from bronchogenic carcinoma. Radiology 1980; 137:49-52.

12 Hendrix RW, Rogers LF, Davis TM, Jr. Cortical bone metastases. Radiology 1991; 181:409-13.

13 Miric A, Banks M, Allen D, et al. Cortical metastatic lesions of the appendicular skeleton from tumors of known primary origin. J Surg Oncol 1998; 67:255-60.

14 Powell GJ, Southby J, Danks JA, et al. Localization of parathyroid hormone-related protein in breast cancer metastases: increased incidence in bone compared with other sites. Cancer Res 1991; 51:3059-61.

15 Basolo F, Fiore L, Fontanini G, et al. Expression of and response to interleukin 6 (IL6) in human mammary tumors. Cancer Res 1996; 56:3118-22.

16 Blay JY, Schemann S, Favrot MC. Local production of interleukin 6 by renal adenocarcinoma in vivo. J Natl Cancer Inst 1994; 86: 238.

17 Tabibzadeh SS, Poubouridis D, May LT, Sehgal PB. Interleukin6 immunoreactivity in human tumors. Am J Pathol 1989; 135:
427-33.

18 Thabard W, Collette M, Mellerin MP, et al. IL-6 upregulates its own receptor on some human myeloma cell lines. Cytokine 2001; 14:352-56.

19 de la Mata J, Uy HL, Guise TA, et al. Interleukin-6 enhances hypercalcemia and bone resorption mediated by parathyroid hormone-related protein in vivo. J Clin Invest 1995; 95:284652.

20 Kurihara N, Bertolini D, Suda T, Akiyama Y, Roodman GD. IL6 stimulates osteoclast-like multinucleated cell formation in long term human marrow cultures by inducing IL-1 release. J Immunol 1990; 144:4226-30.

21 Ono K, Akatsu T, Murakami T, et al. Involvement of cyclooxygenase- 2 in osteoclast formation and bone destruction in bone metastasis of mammary carcinoma cell lines. J Bone Miner Res 2002; 17:774-81.

22 Pederson L, Winding B, Foged NT, Spelsberg TC, Oursler MJ. Identification of breast cancer cell line-derived paracrine factors that stimulate osteoclast activity. Cancer Res 1999; 59:5849-55.

23 Mancino AT, Klimberg VS, Yamamoto M, Manolagas SC, Abe E. Breast cancer increases osteoclastogenesis by secreting MCSF and upregulating RANKL in stromal cells. J Surg Res 2001; 100: $18-24$

24 Sapi E. The role of CSF-1 in normal physiology of mammary gland and breast cancer: an update. Exp Biol Med (Maywood) 2004; 229:1-11.

25 Yin JJ, Selander K, Chirgwin JM, et al. TGF-beta signaling blockade inhibits PTHrP secretion by breast cancer cells and bone metastases development. J Clin Invest 1999; 103:197-206.

26 George J, Lai FM. Metastatic cervical carcinoma presenting as psoas abscess and osteoblastic and lytic bony metastases. Singapore Med J 1995; 36:224-7.

27 Joffe N, Antonioli DA. Osteoblastic bone metastases secondary to adenocarcinoma of the pancreas. Clin Radiol 1978; 29:41-6.

28 Paling MR, Pope TL. Computed tomography of isolated osteoblastic colon metastases in the bony pelvis. J Comput Tomogr 1988; 12:203-7.

29 Mohammad KS, Guise TA. Mechanisms of osteoblastic metastases: role of endothelin-1. Clin Orthop 2003; 415 Suppl: S67-74.

30 Kurihara Y, Kurihara H, Suzuki H, et al. Elevated blood pressure and craniofacial abnormalities in mice deficient in endothelin-1. Nature 1994; 368:703-10.

31 Nelson JB, Hedican SP, George DJ, et al. Identification of endothelin-1 in the pathophysiology of metastatic adenocarcinoma of the prostate. Nat Med 1995; 1:944-9.

32 Yin JJ, Mohammad KS, Kakonen SM, et al. A causal role for endothelin-1 in the pathogenesis of osteoblastic bone metastases. Proc Natl Acad Sci U S A 2003; 100:10954-9.

33 Nelson JB, Chan-Tack K, Hedican SP, et al. Endothelin-1 production and decreased endothelin $\mathrm{B}$ receptor expression in advanced prostate cancer. Cancer Res 1996; 56:663-8.

34 Chiao JW, Moonga BS, Yang YM, et al. Endothelin-1 from prostate cancer cells is enhanced by bone contact which blocks osteoclastic bone resorption. Br J Cancer 2000; 83:360-5.

35 Alam AS, Gallagher A, Shankar V, et al. Endothelin inhibits osteoclastic bone resorption by a direct effect on cell motility: implications for the vascular control of bone resorption. Endocrinology 1992; 130:3617-24. 
36 Lassiter LK, Carducci MA. Endothelin receptor antagonists in the treatment of prostate cancer. Semin Oncol 2003; 30:678-88.

37 Reddi AH. Bone and cartilage differentiation. Curr Opin Genet Dev 1994; 4:737-44.

38 McCarthy TL, Ji C, Chen Y, et al. Runt domain factor (Runx)dependent effects on CCAAT/ enhancer-binding protein delta expression and activity in osteoblasts. J Biol Chem 2000; 275: 21746-53.

39 Thomas BG, Hamdy FC. Bone morphogenetic protein-6: potential mediator of osteoblastic metastases in prostate cancer. Prostate Cancer Prostatic Dis 2000; 3:283-5.

40 Brubaker KD, Corey E, Brown LG, Vessella RL. Bone morphogenetic protein signaling in prostate cancer cell lines. J Cell Biochem 2004; 91:151-60.

41 Ide H, Yoshida T, Matsumoto N, et al. Growth regulation of human prostate cancer cells by bone morphogenetic protein-2. Cancer Res 1997; 57:5022-7.

42 Achbarou A, Kaiser S, Tremblay G, et al. Urokinase overproduction results in increased skeletal metastasis by prostate cancer cells in vivo. Cancer Res 1994; 54:2372-7.

43 Rabbani SA, Desjardins J, Bell AW, et al. An amino-terminal fragment of urokinase isolated from a prostate cancer cell line (PC-3) is mitogenic for osteoblast-like cells. Biochem Biophys Res Commun 1990; 173:1058-64.

44 Killian CS, Corral DA, Kawinski E, Constantine RI. Mitogenic response of osteoblast cells to prostate-specific antigen suggests an activation of latent TGF-beta and a proteolytic modulation of cell adhesion receptors. Biochem Biophys Res Commun 1993; 192:940-7.

45 Yi B, Williams PJ, Niewolna M, Wang Y, and Yoneda T. Tumorderived platelet-derived growth factor-BB plays a critical role in osteosclerotic bone metastasis in an animal model of human breast cancer. Cancer Res 2002; 62:917-23.

46 Oades GM, Coxon J, Colston KW. The potential role of bisphosphonates in prostate cancer. Prostate Cancer Prostatic Dis 2002; 5:264-72.

47 Yonou H, Ochiai A, Goya M, et al. Intraosseous growth of human prostate cancer in implanted adult human bone: relationship of prostate cancer cells to osteoclasts in osteoblastic metastatic lesions. Prostate 2004; 58:406-13.

48 Garnero P, Buchs N, Zekri J, et al. Markers of bone turnover for the management of patients with bone metastases from prostate cancer. Br J Cancer 2000; 82:858-64.

49 Lipton A, Small E, Saad F, et al. The new bisphosphonate, Zometa (zoledronic acid), decreases skeletal complications in both osteolytic and osteoblastic lesions: a comparison to pamidronate. Cancer Invest 2002; 20 Suppl 2:45-54.

50 Powles T, Paterson S, Kanis JA, et al. Randomized, placebocontrolled trial of clodronate in patients with primary operable breast cancer. J Clin Oncol 2002; 20:3219-24. 